\title{
Ancillary Testing in Lung Cancer Diagnosis
}

\author{
William Dubinski, ${ }^{1,2}$ Natasha B. Leighl, ${ }^{3}$ Ming-Sound Tsao, ${ }^{1,2}$ and David M. Hwang ${ }^{1,2}$ \\ ${ }^{1}$ Department of Pathology, Toronto General Hospital, University Health Network, 200 Elizabeth Street, Toronto, \\ ON, Canada M5G 2C4 \\ ${ }^{2}$ Department of Laboratory Medicine and Pathobiology, University of Toronto, 1 King's College Circle, Toronto, \\ ON, Canada M5S 1 A8 \\ ${ }^{3}$ Department of Medical Oncology and Hematology, Princess Margaret Hospital, University Health Network, \\ 610 University Avenue, Toronto, ON, Canada M5G 2M9
}

Correspondence should be addressed to David M. Hwang, david.hwang@uhn.on.ca

Received 15 July 2011; Accepted 5 October 2011

Academic Editor: Joanna Domagala-Kulawik

Copyright ( $) 2012$ William Dubinski et al. This is an open access article distributed under the Creative Commons Attribution License, which permits unrestricted use, distribution, and reproduction in any medium, provided the original work is properly cited.

The pathologic diagnosis of lung cancer historically has relied primarily on morphologic features of tumors in histologic sections. With the emergence of new targeted therapies, the pathologist is called upon increasingly to provide not only accurate typing of lung cancers, but also to provide prognostic and predictive information, based on a growing number of ancillary tests, that may have significant impact on patient management. This review provides an overview of ancillary tests currently used in the pathologic diagnosis of lung cancer, with a focus on immunohistochemistry and molecular diagnostics.

\section{Introduction}

Primary lung cancer has been classified historically into two clinically relevant groups: small cell lung cancer (SCLC) and nonsmall cell lung cancer (NSCLC). This distinction was clinically useful as available treatment strategies differed significantly between these two groups. In recent years, the emerging evidence of differential response to new targeted therapies and the identification of molecular differences between specific subtypes of NSCLC increasingly necessitate greater accuracy in the subtyping of NSCLC.

The current WHO classification of lung cancer [1] has been based almost entirely by assessment of morphologic features using standard hematoxylin and eosin (H\&E) stained sections of tumors. However, a growing number of ancillary studies can help with classification, such as the use of immunohistochemistry (IHC). Beyond simple classification, however, ancillary testing for molecular aberrations is entering routine practice and delivers additional prognostic and predictive information. A new multidisciplinary classification system for primary lung adenocarcinomas has emerged recently [2]. While this system is still based largely on morphology, it moves towards incorporating recent advances in clinical and molecular medicine. In this review, we summarize ancillary tests currently used in the pathologic diagnosis of lung cancer, with a focus on immunohistochemistry and molecular diagnostics.

\section{Immunohistochemistry}

Immunohistochemistry involves the detection and localization of antigens or proteins in tissue sections by the use of antibodies that bind specifically to the antigen of interest. The antibodies are coupled to a detection system which allows them to be visualized in tissue sections. IHC has a range of applications in the practice of pathology and is commonly used by pathologists to help in distinguishing cell types or their origin, using markers that are expressed differentially between different cell types and organs. Additionally, IHC enables one to observe or determine the localization and distribution of various antigens or proteins within the tissue.

However, it needs to be recognized that IHC is neither $100 \%$ specific nor $100 \%$ sensitive. For example, thyroid transcription factor-1 (TTF-1) is widely known as a marker for pulmonary adenocarcinoma but is also highly expressed in 
TABLE 1: Immunohistochemical profiles of squamous cell carcinoma and pulmonary adenocarcinoma.

\begin{tabular}{lcc}
\hline Special stain/IHC & Squamous cell carcinoma & Adenocarcinoma \\
\hline CK7 & $-1+$ & + \\
p63 & + & - \\
CK5/6 & + & - \\
TTF-1 & - & $+/-$ \\
\hline
\end{tabular}

thyroid tumors and may uncommonly also be expressed in carcinomas originating from other primary sites, including, for example, colorectal carcinoma [3]. Morphologic features on H\&E sections remain the basis for diagnosis, and ancillary tests need to be interpreted in the context of the histomorphologic findings. Despite these limitations, it has been shown that a high degree of accuracy in the subtyping of NSCLC can be achieved by applying a simple panel of immunohistochemical markers including TTF-1, tumor protein 63 (P63), cytokeratin (CK) 7, and CK5/6 [4, 5]. Other antibodies such as desmocollin-3 and napsin A have also been recently described as helpful in further refining the subtypes in difficult cases $[6,7]$.

CKs are intermediate filament proteins that provide structural support within the cytoplasm of epithelial cells. Fifty-four CK genes have been identified, and the corresponding keratin proteins are classified by molecular weight and isoelectric $\mathrm{pH}[8]$. In the most general terms, tumors of epithelial origin (termed carcinomas or adenocarcinomas, if gland forming) express CKs, which differentiates them from tumors of mesenchymal origin (sarcomas), hematopoietic origin (lymphomas/leukemias), and melanoma. The various epithelial tissues of the human body also express different CKs, thus different epithelial tumors may often be distinguished based on their unique cytokeratin expression profile.

TTF-1 plays an important role in the embryogenesis of lung, and its expression remains high in type II pneumocytes and Clara cells [9]. TTF-1 has been used as an immunohistochemical marker for primary lung adenocarcinoma, despite recent reports of occasional aberrant TTF-1 staining in tumors from other primary sites (e.g., [3]). TTF-1 is known to regulate the expression of several lung-specific proteins including napsin A, surfactant proteins, and others. Antibodies to napsin A in combination with TTF-1 have been proposed as additional evidence of pulmonary origin of a tumor.

2.1. Squamous Cell Carcinoma. In H\&E stained sections, squamous differentiation is identified by keratinisation and/or formation of intercellular bridges. Both features are specific for squamous cell differentiation and are not seen in other tumor types. While these features are readily observed in well-differentiated tumors, they may be difficult to appreciate or absent in poorly differentiated tumors, especially in small biopsy samples or fine needle aspirate cytology specimens. In such cases, an IHC panel including P63, CK5/6, TTF-1, and CK7 may be helpful, with positive staining for P63 and CK5/6 and concurrent lack of staining for TTF-1 and CK7 supporting squamous differentiation (Table 1) [5].
From a clinical standpoint, it is important to note that squamous differentiation is not evidence of the tumor's site of origin. Metastatic squamous cell tumors to the lung are histologically and immunohistochemically identical to primary lung squamous tumors. At present, there are no immunohistochemical or molecular markers in routine use that reliably differentiate primary pulmonary from metastatic squamous cell carcinomas, as TTF- 1 typically is not expressed in pulmonary squamous cell carcinomas. Therefore, clinical history is crucial to determining the site of origin.

The accurate diagnosis of squamous cell histology has important therapeutic implications. Certain systemic therapy agents are not used in patients with squamous histology for safety or efficacy concerns. Bevacizumab, a monoclonal antibody directed against vascular endothelial growth factor (VEGF) is associated with an increased risk of life threatening pulmonary hemorrhage in patients with squamous cell histology [10]. The association between bleeding complications and this histological type has been seen with other VEGF inhibitors although not all [11, 12]. Also, pemetrexed, a chemotherapeutic agent has been associated with inferior outcomes compared with docetaxel chemotherapy. By contrast, pemetrexed use in the first-, second-line, and maintenance settings has been associated with superior outcomes in patients with nonsquamous histology $[13,14]$. These studies confirm that accurate classification of NSCLC has an important role in patient management and outcomes.

2.2. Adenocarcinoma. The WHO classification [1] of lung tumors has long divided adenocarcinomas primarily into acinar, papillary, solid, bronchioloalveolar (BAC), or mixed subtypes based on histomorphologic features. Classically, adenocarcinomas display gland formation on $\mathrm{H} \& \mathrm{E}$, although subtypes with bronchioloalveolar or solid patterns of growth may lack well-defined glandular structures. The BAC subtype (or adenocarcinoma in situ (AIS) in the new IASLC/ATS/ERS classification [2]) is characterized by exclusively "lepidic" growth pattern, in which neoplastic cells grow along the surfaces of preexisting alveolar structures, without evidence of invasion. Two variants of BAC have been recognized classically, mucinous and nonmucinous. The mucinous variant (mucinous AIS or invasive mucinous adenocarcinoma in the IASLC/ATS/ERS classification [2]) is composed of tall columnar cells with abundant pale cytoplasm that stains positively with histochemical stains for mucin, such as mucicarmine or periodic acid Schiff (PAS). Pulmonary adenocarcinoma is differentiated from squamous cell carcinoma by being typically positive for CK7 and TTF-1, and negative for p63 and CK5/6 (Figure 1). Although most pulmonary adenocarcinomas are positive for TTF-1, a significant subset is negative (15-30\%), especially the mucinous BAC subtype, or those originating in more central locations [15].

Primary lung adenocarcinoma must also be differentiated from adenocarcinoma that has metastasized to lung. Clinical history and IHC can be invaluable in this regard. In particular, the differential expression of CK7 and CK20 may be very useful in characterizing the origin of epithelial neoplasms. Pulmonary adenocarcinoma is typically CK7+/CK20 - by IHC although this cytokeratin profile is 

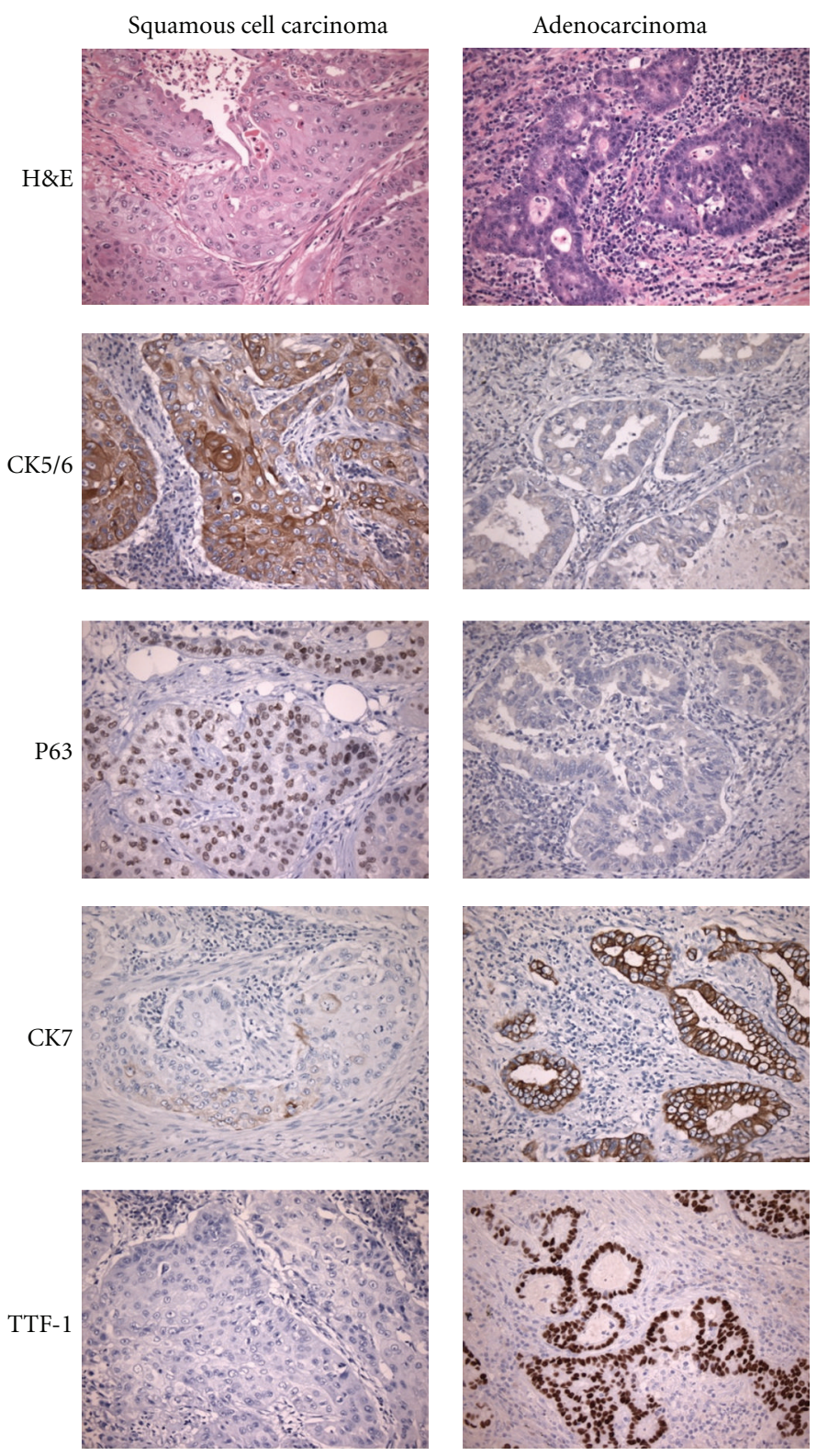

FIGURE 1: Immunohistochemistry stains in squamous cell carcinoma and adenocarcinoma of lung. H\&E: hematoxylin and eosin; CK: cytokeratin; TTF-1: thyroid transcription factor 1. Squamous carcinomas are typically positive for CK5/6 and P63, and negative for CK7 and TTF-1, with the reverse profile for adenocarcinoma although this case of squamous cell carcinoma demonstrates focal weak staining for CK7.

not specific to adenocarcinomas of lung and may be seen also in tumors arising from the breast, thyroid, upper gastrointestinal and pancreaticobiliary tracts, and gynecologic tract. Tumors demonstrating CK7-/CK20+ staining profile include colorectal and Merkel cell carcinomas. CK20 staining may occasionally be seen in pulmonary adenocarcinomas, but CK7 typically is also positive in such cases. While an exhaustive discussion is beyond the scope of this manuscript, suffice to indicate that most primary lung adenocarcinomas are CK7 and TTF-1 positive, although as previously noted, TTF-1 staining may also be seen in thyroid tumors and infrequently in tumors originating from other body sites. Lack of TTF-1 expression does not exclude pulmonary origin for a CK7-positive adenocarcinoma in the lung; however, in this situation, metastatic carcinoma arising from other body sites that demonstrate a $\mathrm{CK} 7+/ \mathrm{CK} 20$ - cytokeratin profile would need to be excluded clinically.

Napsin A, an aspartic acid protease whose expression in the lung is regulated by TTF-1, has also shown promise in helping to differentiate primary lung from metastatic adenocarcinomas. While napsin A expression may also be seen in normal kidney and in a proportion of renal tumors, positivity for both TTF- 1 and napsin A is a strong indication that an adenocarcinoma originated from lung $[6,16]$.

2.3. Neuroendocrine Tumors. Tumors with neuroendocrine (NE) differentiation include small cell carcinoma, large cell neuroendocrine carcinoma, and typical and atypical 
TABLE 2: Immunohistochemical profile of neuroendocrine tumors.

\begin{tabular}{lcccc}
\hline IHC & Typical carcinoid & Atypical carcinoid & Primary lung small cell ca. & Metastatic small cell ca. \\
\hline CD56 & + & + & + & + \\
Synaptophysin & + & + & $-/+$ & $+/-$ \\
Chromogranin & + & + & Usually + & $-/+$ \\
TTF-1 & $-/+$ & $-/+$ & $>20 \%$ (typically $>60 \%)$ & $>20 \%$ (typically $>60 \%)$ \\
Ki-67 & $0-5 \%$ & $5-15 \%$ & &
\end{tabular}

carcinoid tumors. NE tumors are defined ultrastructurally by having neurosecretory granules and immunohistochemically by positivity for NE markers (Table 2). According to a recent international workshop on pulmonary NE tumors, CD56 has a sensitivity of $95 \%$ and a specificity of $97 \%$ for detecting cells showing NE differentiation. The sensitivity for synaptophysin and chromogranin is $80-85 \%$, and the specificity for both is $97 \%$ [17]. Using a panel of two or three NE markers ensures a high degree of accuracy for detecting NE differentiation.

Differentiating between the different types of NE tumors may be very difficult in small biopsy specimens because the small sample may not be entirely representative of the overall tumor and is subject to crush artefact because of the relative fragility of these cells. In some cases, the evaluation of Ki67 may be useful in this regard. Ki-67 is a marker of cell proliferation, and a recent review has concluded that the Ki-67 proliferation index of typical carcinoid (TC) is less than 2\%, while atypical carcinoid (AC) is less than $20 \%$ (typically around 10\%) [18]. Small cell carcinomas often have a Ki-67 proliferation index of greater than $60 \%$. It has been suggested that a Ki-67 index of less than $25 \%$ excludes small cell carcinoma [19].

Differentiating primary NE tumors of the lung from NE tumors from other body sites may also be a challenge, as NE tumors arising from other body sites may demonstrate identical morphology. In this regard, expression of TTF-1 may be helpful to identify a carcinoid tumor of lung origin, as carcinoid tumors from other sites rarely express this marker [20]. However, small cell carcinomas arising from the lung frequently express TTF-1 while those arising from other sites may occasionally also express TTF-1. In the case of small cell carcinoma with TTF-1 positivity, lung origin is favored, but this is not entirely definitive [21].

\subsection{Other IHC Markers}

P53. P53 functions as a tumor suppressor protein by playing a key role in cell cycle progression, apoptosis, and DNA repair [22]. Overexpression of P53 in NSCLC has been reported to be both a prognostic marker, associated with increased tumor aggressiveness and shorter overall survival, and a potential predictive marker, associated with a favorable response to platinum-based adjuvant chemotherapy, resulting in a survival benefit [23]. However, this needs additional validation.
ASH1. From an embryologic perspective, there is now evidence that the transcription factor achaete-scute homologue1 (ASH1) is pivotal for NE cell differentiation and may be necessary for transformation to NE lung carcinoma [24]. Immunohistochemical analysis of ASH1 is available but not routinely used outside of the research setting at present. It may potentially help to identify early progenitor cells that are committed to NE differentiation and better define subsets of lung cancers demonstrating NE differentiation [24].

\section{Molecular Diagnostics in NSCLC}

Molecular testing of lung cancers has seen significant advances in recent years. The potential information derived from molecular tests is enormous and has increasing applications in clinical settings. In the setting of lung cancer, molecular techniques currently employed include both polymerase chain reaction- (PCR) based tests and fluorescent in-situ hybridization (FISH). Both of these tests can be done using formalin-fixed-paraffin-embedded (FFPE) tissue provided that there is sufficient tumor quantity and a nondegraded specimen. PCR is a molecular technique used to amplify short segments of DNA. The amplified DNA from tumor samples can then be subject to various analyses to identify differences (mutations) from a "normal" control. FISH is a technique whereby fluorescent labeled probes are admixed with a test sample and the probes bind to specific DNA sequences if present. Fluorescent microscopy is then used to analyze if and where on the chromosomes the probes have bound. Probes can be designed to bind either normal segments of DNA or segments with known aberrations. A brief review of selected genetic abnormalities with clinical relevance in lung cancer follows (summarized in Table 3). A more extensive review of molecular predictive and prognostic markers has been published elsewhere [25].

3.1. Epidermal Growth Factor Receptor (EGFR). EGFR represents a family of transmembrane tyrosine kinase growth factor receptors involved in a wide array of cellular processes, including proliferation, apoptosis, angiogenesis, and others. A number of patients with NSCLC have dysregulated EGFR resulting in overexpression, amplification, or mutations [26]. Clinical and pathological features associated with high-frequency mutations in the EGFR tyrosine kinase (TK) domain in NSCLC include East Asian ethnicity, females, light/never smokers, and tumors with adenocarcinoma histology. In the general North American or European population, approximately 10-17\% of NSCLC cases may have EGFR 
TABLE 3: Clinically relevant molecular and clinicopathologic features of NSCLC.

\begin{tabular}{lccc}
\hline & EGFR mutation & K-ras mutation & EML4-ALK mutation \\
\hline Histologic subtype & Adenocarcinoma & NSCLC (Adeno > squamous) & $\begin{array}{c}\text { Adenocarcinoma, signet ring } \\
\text { morphology } \\
\text { Clinical features }\end{array}$ \\
Asian, female, light/never smokers & Past or present smokers & $\begin{array}{c}\text { Light/never smokers, possible } \\
\text { young age and male }\end{array}$ & $\begin{array}{c}\text { Use of ALK inhibitors } \\
\text { (currently under study) }\end{array}$ \\
& Sensitive to EGFR TKI therapy & therapy, possible poor prognostic \\
& marker & &
\end{tabular}

mutations [27]. In light or never-smoking Asian patients with adenocarcinoma, the rate of EGFR mutations may be as high as $60 \%$ [28]. Mutated EGFR predicts for high response rate to EGFR TK inhibitor (TKI) therapy, a firstline treatment choice in advanced NSCLC patient population [29], with better progression-free survival and quality of life. Accurate mutation analysis is also important because progression-free survival is shorter if EGFR wild-type patients are treated with EGFR TKIs as opposed to conventional chemotherapy. There is also evidence that different mutations may have differential response rates and relative resistance to EGFR TKI's [30].

Aberrations in EGFR are common in a wide variety of human cancers, but the site of mutation varies with the type of malignancy. Activating mutations in EGFR result in constitutively activated downstream signalling pathways. Studies on EGFR mutations in NSCLC show that approximately 90\% occur within exons 18-21 [31]. The specific type of mutation may hold predictive relevance in that some mutations confer higher affinity for certain EGFR TKIs and some confer resistance to certain EGFR TKIs, for example, mutations in exon 20 and the T790M mutation. Various different methods are currently used for the detection of EGFR mutations (reviewed in [32]). Of these, direct sequencing of PCR amplicons from the EGFR gene is perhaps the most widely used but is also cost and labor intensive. Targeted detection of specific mutations is also an option, given that two mutations account for more than $90 \%$ of all EGFR mutations in NSCLC (exon 19 deletions and the exon 21 L858R mutation) [33]. However, the development of monoclonal antibodies directed against the two most common mutations may enable immunohistochemical screening for EGFR mutations, providing rapid results at a fraction of the cost of current molecular testing [34].

3.2. K-RAS. The ras family of oncogenes encode for proteins that mediate signalling pathways controlling cell growth. There are three distinct ras genes (H-ras, K-ras, $\mathrm{N}$-ras), each of which has been associated with various malignancies. In NSCLC, $>90 \%$ of ras mutations occur on the K-ras gene, which would result in the constitutive activation of downstream signalling pathways. K-ras mutations are seen almost exclusively in smokers, and this relationship does not seem to be dose or time dependent [35]. K-ras mutations in NSCLC occur mainly in adenocarcinoma. As a prognostic marker, K-ras mutation appears to be associated weakly with poorer outcome [36]. K-ras mutations rarely occur in tumors with EGFR mutations. Their presence has been associated with relative resistance to EGFR TKI therapy [37] although currently patients with K-ras mutations are not excluded from EGFR TKI therapy. In the rare patient that has both a K-ras mutation and an activating EGFR mutation, EGFR TKI therapy may be appropriate $[38,39]$.

3.3. EML4-ALK. The anaplastic lymphoma kinase (ALK) gene was originally identified as part of a chromosomal translocation found in a subset of anaplastic large cell lymphomas. Subsequently, it has been shown that ALK is dysregulated in a number of solid and hematologic malignancies. The mechanisms of dysregulation include translocations as well as mutations in the tyrosine kinase domain [40]. In 2007, a translocation between echinoderm microtubule-associated protein-like 4 (EML4) and ALK was identified in a subset of lung adenocarcinomas [41]. The fusion product has oncogenic properties, and transgenic mice that express EML4-ALK in alveoli develop hundreds of nodules of adenocarcinoma [42].

The result of an early-phase trial with crizotinib, an inhibitor developed for ALK and Met, has generated considerable interest as a therapeutic option in patients with NSCLC who harbor EML4-ALK mutations [43]; a number of clinical trials are currently underway to determine their efficacy and parameters for optimal use.

The overall incidence of EML4-ALK rearrangements in NSCLC appears to be approximately 3.5\% [44]. There appears to be a similar frequency of ALK mutations across different ethnicities, suggesting that race may not be as relevant as for EGFR mutations. Clinical parameters associated with ALK mutations include never/light smokers, and in some studies there is an association with younger patient age and male sex $[45,46]$. From a histologic perspective, EML4-ALK rearrangement is seen almost exclusively in adenocarcinomas, and only rare cases have been reported in squamous cell carcinoma $[41,46]$. Interestingly, signet ring cell histology has been associated frequently with ALK rearranged tumors [45].

A variety of techniques are available to detect ALK gene rearrangements including FISH, IHC, and PCR. FISH probes that identify ALK rearrangements are commonly used in clinical trials. The limitation of this method is that the translocation can be difficult to detect due to the small size of the inversion [47]. Attempts to use IHC to detect ALK-rearranged tumors initially met with limited success as the fusion protein is expressed at low levels; 
the development of novel specific and sensitive antibodies, however, seems on the horizon [48]. PCR-based techniques have not gained widespread use as PCR-based detection of the fusion transcript is generally not optimal in FFPE tissue. The optimal method for identifying EML4-ALK fusion is yet to be definitively determined [49] although FISH is widely viewed as the current gold standard.

3.4. MET. MET is a receptor tyrosine kinase involved in important signalling pathways related to cell proliferation, angiogenesis, and tumor aggression [50]. MET signalling becomes dysregulated in both NSCLC and small cell lung cancer through various mechanisms, including overexpression, mutations, amplifications, and autocrine/paracrine activation of hepatocyte growth factor [51]. MET gene amplification as detected by fluorescence in situ hybridization may be a poor prognostic factor in NSCLC [52]. MET amplification has also been implicated as one mechanism for acquired resistance to EGFR TKI therapy and may be seen in 5 to $20 \%$ of resistant cases $[53,54]$. As a therapeutic option, MET inhibitors alone or in combination with EGFR TKIs are in clinical trials.

\section{Conclusion}

Clinical, pathological, and genetic parameters are beginning to converge in NSCLC. Selection of patients based on clinical and histopathologic criteria can, for example, considerably increase the likelihood of identifying EML4-ALK and EGFR mutations. In addition, the development of treatment protocols directed at specific molecularly or histologically defined subsets of NSCLC has improved treatment outcomes for patients. The role of the pathologist is now to provide not only accurate subtyping of lung cancers, but increasingly also to provide prognostic and predictive information that is critical to patient outcomes. Future developments will continue to refine our understanding and approach to lung cancer. Several lung cancer studies have used microarray platforms to measure the expression of thousands of genes simultaneously. The results generated provide unique gene-expression profiles with significant prognostic and predictive impact $[55,56]$. Gene expression profiling may also help refine tumor classification. While these and other molecular profiling strategies need further validation in prospective studies, currently available technology points to a future where the molecular profiling will increasingly help guide classification, treatment, and prognosis of lung cancers.

\section{References}

[1] W. D. Travis, E. Brambilla, H. K. Muller-Hermelink, and C. C. Harris, Pathology and Genetics of Tumours of the Lung, Pleura, Thymus, and Heart, World Health Organization Classification of Tumours, IARC, Lyon, France, 2004.

[2] W. D. Travis, E. Brambilla, M. Noguchi et al., "International association for the study of lung cancer/American Thoracic Society/European Respiratory Society international multidisciplinary classification of lung adenocarcinoma," Journal of Thoracic Oncology, vol. 6, no. 2, pp. 244-285, 2011.
[3] B. Xu, N. Thong, D. Tan, and T. Khoury, "Expression of thyroid transcription factor-1 in colorectal carcinoma," Applied Immunohistochemistry and Molecular Morphology, vol. 18, no. 3, pp. 244-249, 2010.

[4] P. S. Loo, S. C. Thomas, M. C. Nicolson, M. N. Fyfe, and K. M. Kerr, "Subtyping of undifferentiated non-small cell carcinomas in bronchial biopsy specimens," Journal of Thoracic Oncology, vol. 5, no. 4, pp. 442-447, 2010.

[5] A. G. Nicholson, D. Gonzalez, P. Shah et al., "Refining the diagnosis and EGFR status of non-small cell lung carcinoma in biopsy and cytologic material, using a panel of Mucin staining, TTF-1, cytokeratin 5/6, and P63, and EGFR mutation analysis," Journal of Thoracic Oncology, vol. 5, no. 4, pp. 436$441,2010$.

[6] J. Ye, J. J. Findeis-Hosey, Q. Yang et al., "Combination of napsin A and TTF-1 immunohistochemistry helps in differentiating primary lung adenocarcinoma from metastatic carcinoma in the lung," Applied Immunohistochemistry and Molecular Morphology, vol. 19, no. 4, pp. 313-317, 2011.

[7] V. Monica, P. Ceppi, L. Righi et al., "Desmocollin-3: a new marker of squamous differentiation in undifferentiated largecell carcinoma of the lung," Modern Pathology, vol. 22, no. 5, pp. 709-717, 2009.

[8] J. Schweizer, P. E. Bowden, P. A. Coulombe et al., "New consensus nomenclature for mammalian keratins," Journal of Cell Biology, vol. 174, no. 2, pp. 169-174, 2006.

[9] V. Boggaram, "Thyroid transcription factor-I (TTFI/Nkx2.I/TITFI) gene regulation in the lung," Clinical Science, vol. 116, no. 1, pp. 27-35, 2009.

[10] D. H. Johnson, L. Fehrenbacher, W. F. Novotny et al., "Randomized phase II trial comparing bevacizumab plus carboplatin and paclitaxel with carboplatin and paclitaxel alone in previously untreated locally advanced or metastatic nonsmall-cell lung cancer," Journal of Clinical Oncology, vol. 22, no. 11, pp. 2184-2191, 2004.

[11] A. B. Sandler, J. H. Schiller, R. Gray et al., "Retrospective evaluation of the clinical and radiographic risk factors associated with severe pulmonary hemorrhage in first-line advanced, unresectable non-small-cell lung cancer treated with carboplatin and paclitaxel plus bevacizumab," Journal of Clinical Oncology, vol. 27, no. 9, pp. 1405-1412, 2009.

[12] M. A. Socinski, S. Novello, J. R. Brahmer et al., "Multicenter, phase II trial of sunitinib in previously treated, advanced nonsmall-cell lung cancer," Journal of Clinical Oncology, vol. 26, no. 4, pp. 650-656, 2008.

[13] G. V. Scagliotti, P. Parikh, J. Von Pawel et al., "Phase III study comparing cisplatin plus gemcitabine with cisplatin plus pemetrexed in chemotherapy-naive patients with advancedstage non-small-cell lung cancer," Journal of Clinical Oncology, vol. 26, no. 21, pp. 3543-3551, 2008.

[14] T. Ciuleanu, T. Brodowicz, C. Zielinski et al., "Maintenance pemetrexed plus best supportive care versus placebo plus best supportive care for non-small-cell lung cancer: a randomised, double-blind, phase 3 study," The Lancet, vol. 374, no. 9699, pp. 1432-1440, 2009.

[15] Y. Yatabe, T. Mitsudomi, and T. Takahashi, "TTF-1 expression in pulmonary adenocarcinomas," American Journal of Surgical Pathology, vol. 26, no. 6, pp. 767-773, 2002.

[16] N. Fatima, C. Cohen, D. Lawson, and M. T. Siddiqui, "TTF1 and Napsin A double stain: a useful marker for diagnosing lung adenocarcinoma on fine-needle aspiration cell blocks," Cancer Cytopathology, vol. 119, no. 2, pp. 127-133, 2011. 
[17] E. Lim, P. Goldstraw, A. G. Nicholson et al., "Proceedings of the IASLC international workshop on advances in pulmonary neuroendocrine tumors 2007," Journal of Thoracic Oncology, vol. 3, no. 10, pp. 1194-1201, 2008.

[18] N. Rekhtman, "Neuroendocrine tumors of the lung: an update," Archives of Pathology and Laboratory Medicine, vol. 134, no. 11 , pp. 1628-1638, 2010.

[19] D. L. Asian, H. E. Gulbahce, S. E. Pambuccian, J. C. Manivel, and J. Jessurun, "Ki-67 immunoreactivity in the differential diagnosis of pulmonary neuroendocrine neoplasms in specimens with extensive crush artifact," American Journal of Clinical Pathology, vol. 123, no. 6, pp. 874-878, 2005.

[20] E. Z. Du, P. Goldstraw, J. Zacharias et al., "TTF-1 expression is specific for lung primary in typical and atypical carcinoids: TTF-1-positive carcinoids are predominantly in peripheral location," Human Pathology, vol. 35, no. 7, pp. 825-831, 2004.

[21] O. Kaufmann and M. Dietel, "Expression of thyroid transcription factor- 1 in pulmonary and extrapulmonary small cell carcinomas and other neuroendocrine carcinomas of various primary sites," Histopathology, vol. 36, no. 5, pp. 415-420, 2000.

[22] W. S. El-Deiry, "Review: the p53 pathway and cancer therapy," The Cancer Journal, vol. 11, pp. 229-236, 1998.

[23] M. S. Tsao, S. Aviel-Ronen, K. Ding et al., "Prognostic and predictive importance of $\mathrm{p} 53$ and RAS for adjuvant chemotherapy in non-small-cell lung cancer," Journal of Clinical Oncology, vol. 25, no. 33, pp. 5240-5247, 2007.

[24] M. Miki, D. W. Ball, and R. I. Linnoila, "Insights into the achaete-scute homolog-1 gene (hASH1) in normal and neoplastic human lung," Lung Cancer, vol. 75, no. 1, pp. 58-65, 2012.

[25] L. E. Coate, T. John, M. S. Tsao, and F. A. Shepherd, "Molecular predictive and prognostic markers in non-small-cell lung cancer," The Lancet Oncology, vol. 10, no. 10, pp. 1001-1010, 2009.

[26] F. R. Hirsch, M. Varella-Garcia, P. A. Bunn Jr. et al., "Epidermal growth factor receptor in non-small-cell lung carcinomas: correlation between gene copy number and protein expression and impact on prognosis," Journal of Clinical Oncology, vol. 21, no. 20, pp. 3798-3807, 2003.

[27] R. Rosell, T. Moran, C. Queralt et al., "Screening for epidermal growth factor receptor mutations in lung cancer," New England Journal of Medicine, vol. 361, no. 10, pp. 958-967, 2009.

[28] T. S. Mok, Y. L. Wu, S. Thongprasert et al., "Gefitinib or carboplatin-paclitaxel in pulmonary adenocarcinoma," New England Journal of Medicine, vol. 361, no. 10, pp. 947-957, 2009.

[29] P. A. Bunn, R. Dziadziuszko, M. Varella-Garcia et al., "Biological markers for non-small cell lung cancer patient selection for epidermal growth factor receptor tyrosine kinase inhibitor therapy," Clinical Cancer Research, vol. 12, no. 12, pp. 36523656, 2006.

[30] D. M. Jackman, V. A. Miller, L. A. Cioffredi et al., "Impact of epidermal growth factor receptor and KRAS mutations on clinical outcomes in previously untreated non-small cell lung cancer patients: results of an online tumor registry of clinical trials," Clinical Cancer Research, vol. 15, no. 16, pp. 5267-5273, 2009.

[31] G. J. Riely, K. A. Politi, V. A. Miller, and W. Pao, "Update on epidermal growth factor receptor mutations in non-small cell lung cancer," Clinical Cancer Research, vol. 12, no. 24, pp. 7232-7241, 2006.
[32] T. John, G. Liu, and M. S. Tsao, "Overview of molecular testing in non-small-cell lung cancer: mutational analysis, gene copy number, protein expression and other biomarkers of EGFR for the prediction of response to tyrosine kinase inhibitors," Oncogene, vol. 28, supplement 1, pp. S14-S23, 2009.

[33] H. Shigematsu, L. Lin, T. Takahashi et al., "Clinical and biological features associated with epidermal growth factor receptor gene mutations in lung cancers," Journal of the National Cancer Institute, vol. 97, no. 5, pp. 339-346, 2005.

[34] J. Yu, S. Kane, J. Wu et al., "Mutation-specific antibodies for the detection of EGFR mutations in non-small-cell lung cancer," Clinical Cancer Research, vol. 15, no. 9, pp. 3023-3028, 2009.

[35] S. A. Ahrendt, P. Anthony Decker, E. A. Alawi et al., "Cigarette smoking is strongly associated with mutation of the K-ras gene in patients with primary adenocarcinoma of the lung," Cancer, vol. 92, no. 6, pp. 1525-1530, 2001.

[36] C. Mascaux, N. Iannino, B. Martin et al., "The role of RAS oncogene in survival of patients with lung cancer: a systematic review of the literature with meta-analysis," British Journal of Cancer, vol. 92, no. 1, pp. 131-139, 2005.

[37] K. Suda, K. Tomizawa, and T. Mitsudomi, "Biological and clinical significance of KRAS mutations in lung cancer: an oncogenic driver that contrasts with EGFR mutation," Cancer and Metastasis Reviews, vol. 29, no. 1, pp. 49-60, 2010.

[38] D. M. Jackman, V. A. Miller, L. A. Cioffredi et al., "Impact of epidermal growth factor receptor and KRAS mutations on clinical outcomes in previously untreated non-small cell lung cancer patients: results of an online tumor registry of clinical trials," Clinical Cancer Research, vol. 15, no. 16, pp. 5267-5273, 2009.

[39] A. Sakurada and M. S. Tsao, "Predictive biomarkers for EGFR therapy," IDrugs, vol. 12, no. 1, pp. 34-38, 2009.

[40] Y. P. Mossé, A. Wood, and J. M. Maris, "Inhibition of ALK signaling for cancer therapy," Clinical Cancer Research, vol. 15, no. 18, pp. 5609-5614, 2009.

[41] M. Soda, Y. L. Choi, M. Enomoto et al., "Identification of the transforming EML4-ALK fusion gene in non-small-cell lung cancer," Nature, vol. 448, no. 7153, pp. 561-566, 2007.

[42] M. Soda, S. Takada, K. Takeuchi et al., "A mouse model for EML4-ALK-positive lung cancer," Proceedings of the National Academy of Sciences of the United States of America, vol. 105, no. 50, pp. 19893-19897, 2008.

[43] E. L. Kwak, Y. J. Bang, D. R. Camidge et al., "Anaplastic lymphoma kinase inhibition in non-small-cell lung cancer," New England Journal of Medicine, vol. 363, no. 18, pp. 1693-1703, 2010.

[44] B. Solomon, M. Varella-Garcia, and D. R. Camidge, "ALK gene rearrangements: a new therapeutic target in a molecularly defined subset of non-small cell lung cancer," Journal of Thoracic Oncology, vol. 4, no. 12, pp. 1450-1454, 2009.

[45] S. J. Rodig, M. Mino-Kenudson, S. Dacic et al., "Unique clinicopathologic features characterize ALK-rearranged lung adenocarcinoma in the western population," Clinical Cancer Research, vol. 15, no. 16, pp. 5216-5223, 2009.

[46] A. T. Shaw, B. Y. Yeap, M. Mino-Kenudson et al., "Clinical features and outcome of patients with non-small-cell lung cancer who harbor EML4-ALK," Journal of Clinical Oncology, vol. 27, no. 26, pp. 4247-4253, 2009.

[47] T. Sasaki, S. J. Rodig, L. R. Chirieac, and P. A. Jänne, “The biology and treatment of EML4-ALK non-small cell lung cancer," European Journal of Cancer, vol. 46, no. 10, pp. 1773-1780, 2010. 
[48] M. Mino-Kenudson, L. R. Chirieac, K. Law et al., "A novel, highly sensitive antibody allows for the routine detection of ALK-rearranged lung adenocarcinomas by standard immunohistochemistry," Clinical Cancer Research, vol. 16, no. 5, pp. 1561-1571, 2010.

[49] W. A. Cooper, S. O’toole, M. Boyer, L. Horvath, and A. Mahar, "What's new in non-small cell lung cancer forpathologists: the importance of accurate subtyping, EGFR mutations and ALK rearrangements," Pathology, vol. 43, no. 2, pp. 103-115, 2011.

[50] J. G. Christensen, J. Burrows, and R. Salgia, "c-Met as a target for human cancer and characterization of inhibitors for therapeutic intervention," Cancer Letters, vol. 225, no. 1, pp. 1-26, 2005.

[51] E. S. Kim and R. Salgia, "MET pathway as a therapeutic target," Journal of Thoracic Oncology, vol. 4, no. 4, pp. 444-447, 2009.

[52] F. Cappuzzo, A. Marchetti, M. Skokan et al., "Increased MET gene copy number negatively affects survival of surgically resected non-small-cell lung cancer patients," Journal of Clinical Oncology, vol. 27, no. 10, pp. 1667-1674, 2009.

[53] J. A. Engelman, K. Zejnullahu, T. Mitsudomi et al., "MET amplification leads to gefitinib resistance in lung cancer by acti vating ERBB3 signaling," Science, vol. 316, no. 5827, pp. 10391043, 2007.

[54] L. V. Sequist, B. A. Waltman, D. Dias-Santagata et al., "Genotypic and histological evolution of lung cancers acquiring resistance to EGFR inhibitors," Science Translational Medicine, vol. 3, no. 75, 2011.

[55] H. Kadara, C. Behrens, P. Yuan et al., "A five-gene and corresponding protein signature for stage-I lung adenocarcinoma prognosis," Clinical Cancer Research, vol. 17, no. 6, pp. 14901501, 2011.

[56] H. Y. Chen, S. L. Yu, C. H. Chen et al., "A five-gene signature and clinical outcome in non-small-cell lung cancer," New England Journal of Medicine, vol. 356, no. 1, pp. 11-20, 2007. 


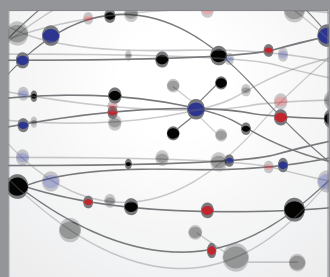

The Scientific World Journal
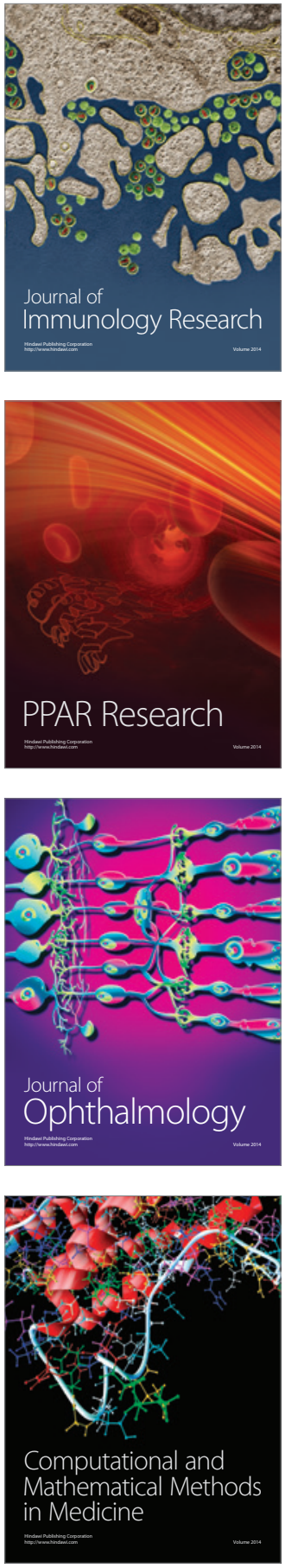

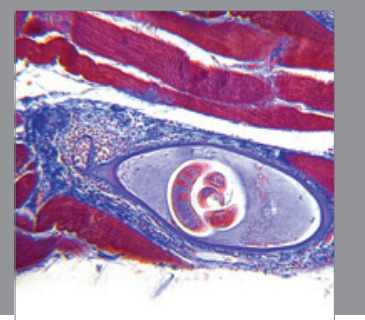

Gastroenterology

Research and Practice
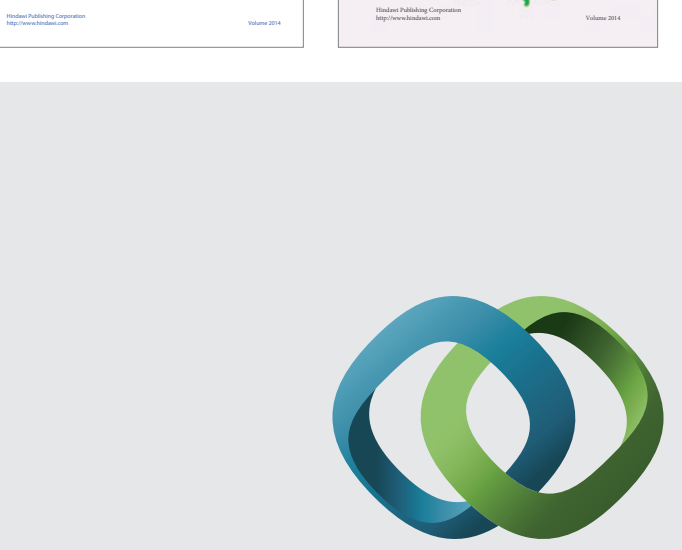

\section{Hindawi}

Submit your manuscripts at

http://www.hindawi.com
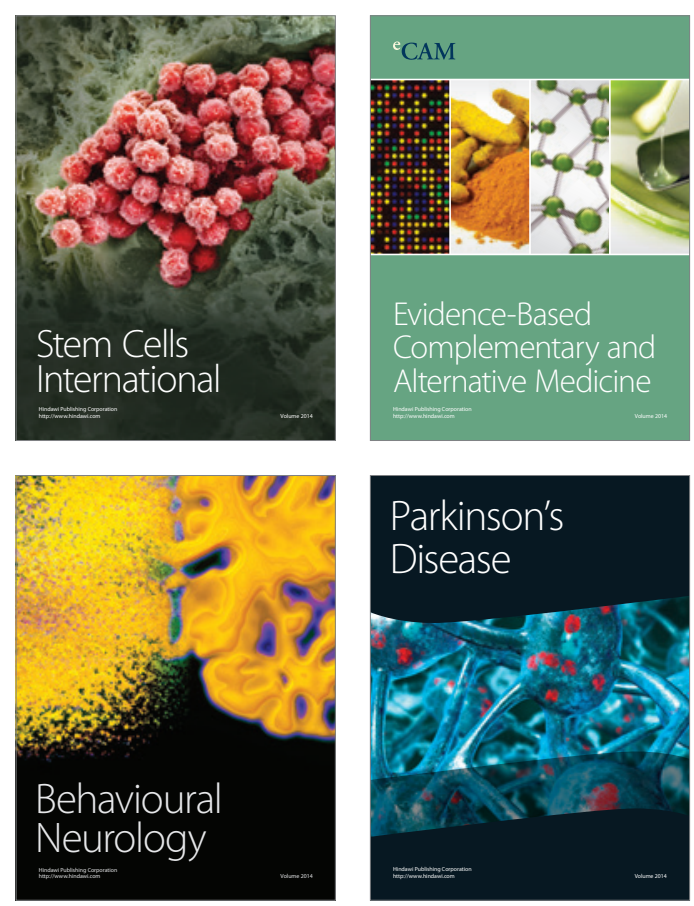

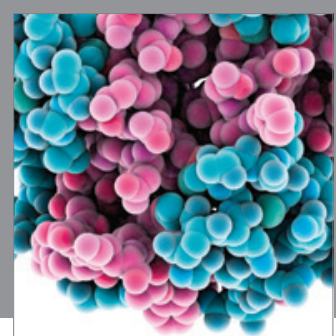

Journal of
Diabetes Research

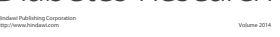

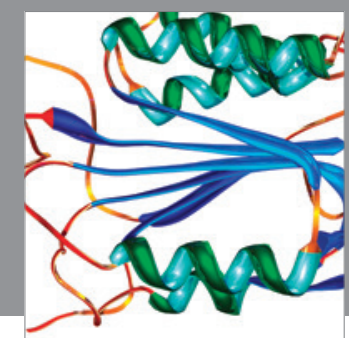

Disease Markers
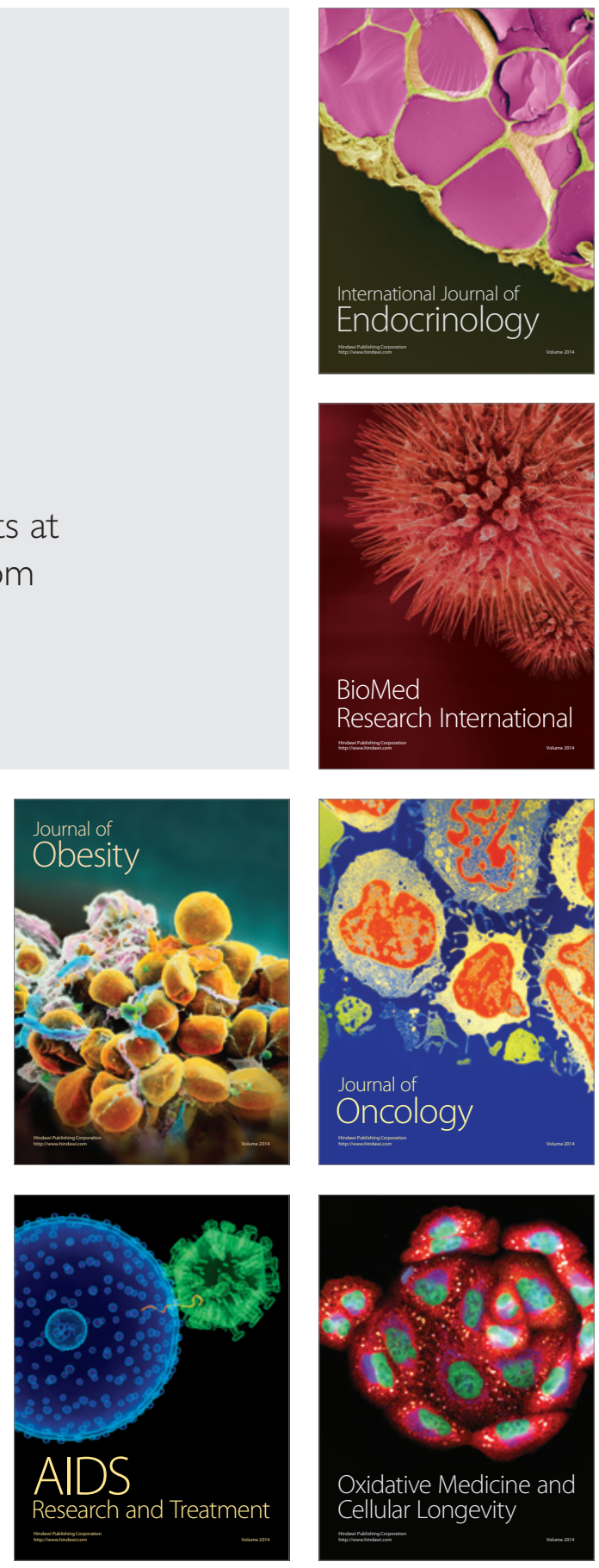